\section{Controlling the HIV Epidemic in Canada: A Public Health Success?}

HIV is among the most important public health challenges we face in Canada. Yet, 35 years into the epidemic, it is clear we have not fared well. Data on HIV incidence and prevalence reveal our lack of success in preventing and controlling this infection. We appear to be experiencing more rapid HIV spread compared to the United States, the natural referent for Canada. In 1998, HIV prevalence in the US was 2.2 times higher than in Canada, but in 2009, only 1.8 times higher. Furthermore, in 2009 there were 4.7 incident infections for every 100 prevalent infections in Canada, compared to 4.1 in the US (i.e., $14 \%$ higher).

The picture is mixed for the two populations most affected by HIV. For injecting drug users, we have reduced or maintained low HIV incidence in the three major urban centres where this activity is concentrated, likely thanks to enlightened and aggressive policies of needle exchange, drug rehabilitation programs and, in some centres, supervised injection sites. For men who have sex with men (MSM), we have not done as well. In 2011, 1,500 MSM were newly infected compared to less than 900 in 1999, an increase of 70\%. In fairness, many other Western countries have also failed to control HIV in this population.

Several factors contribute to our lack of success. Despite the commitment of federal government funds to HIV prevention and research, our overall scorecard is mediocre at best. The Public Health Agency of Canada (PHAC), established to provide independent leadership in public health, has failed to deliver. Efforts to monitor the epidemic have been exemplary but leadership in HIV prevention policy and research has been sorely lacking. No leaders at the federal level have emerged with the training, experience and special skills to coordinate our efforts and develop innovative and effective strategies to control HIV infection. Though the Canadian Institutes of Health Research (CIHR) supports some HIV prevention research, this has been relatively limited. The resources for effective, and sometimes even cost-effective, strategies have also been inadequate.

We compare poorly to the US Centers for Disease Control, where well-trained and experienced personnel have blazed trails to deal with the HIV epidemic. In 2010, the US released their impressive strategy which included objective and quantifiable measures of impact with timelines. It is often claimed that PHAC's role is limited because our constitution relegates responsibility for health to the provinces. In fact, in the US, health is also primarily a state jurisdiction. The Canadian government is responsible for leading, coordinating, and supporting health problems of national scope. HIV certainly qualifies in this regard.

We need to mobilize Canada's best minds at the federal level. We need a team of competent and experienced public health specialists, epidemiologists, social scientists and economists to develop and support innovative and effective policies and programs to deal effectively with the HIV epidemic.

Robert Remis

Interim Scientific Editor

\section{La lutte contre l'épidémie de VIH au Canada : une victoire pour la santé publique?}

Le VIH compte parmi les plus grands problèmes de santé publique au Canada. Pourtant, 35 ans après le début de l'épidémie, il est clair que nous n'en sortons pas. Les données sur l'incidence et la prévalence du VIH montrent que nous ne réussissons ni à prévenir, ni à maîtriser l'infection. La propagation du VIH semble plus rapide ici qu'aux États-Unis, le point de référence naturel pour le Canada. En 1998, la prévalence du VIH aux États-Unis était 2,2 fois plus élevée qu'au Canada, mais en 2009, elle n'était plus qu'1,8 fois plus élevée. De plus, en 2009 il y a eu au Canada 4,7 infections incidentes pour 100 infections existantes, contre 4,1 aux États-Unis (soit $14 \%$ de plus).

Le portrait est mitigé pour les deux populations les plus touchées par le VIH. Chez les utilisateurs de drogue par injection, nous avons réduit ou maintenu à un faible niveau l'incidence du VIH dans les trois grands centres urbains où cette activité est concentrée, probablement grâce aux politiques éclairées et musclées d'échange de seringues, aux programmes de traitement de la toxicomanie et, dans certains centres, aux sites d'injection supervisée. Pour les hommes ayant des relations sexuelles avec des hommes (HARSAH), nous n'avons pas fait aussi bien. En 2011, $1500 \mathrm{HARSAH}$ ont été nouvellement infectés, contre moins de 900 en 1999, soit une hausse de $70 \%$. En toute justice, beaucoup d'autres pays occidentaux ne réussissent pas non plus à endiguer le VIH dans cette population.

Plusieurs facteurs contribuent à notre insuccès. Malgré l'engagement de fonds fédéraux dans la prévention et la recherche sur le VIH, notre fiche de pointage générale est, au mieux, médiocre. L'Agence de la santé publique du Canada, créée pour assurer un leadership indépendant en santé publique, n'a pas tenu sa promesse. Ses efforts de surveillance de l'épidémie sont exemplaires, mais son leadership au chapitre des politiques et de la recherche en prévention du VIH est nettement insuffisant. Aucun chef de file, au palier fédéral, ne semble avoir la formation, l'expérience et les compétences spéciales qu'il faut pour coordonner notre action et élaborer des stratégies novatrices et efficaces de lutte contre l'infection à VIH. Les Instituts de recherche en santé du Canada (IRSC) appuient bien quelques études en prévention du VIH, mais dans une mesure limitée. Les ressources nécessaires à des stratégies efficaces, et même parfois rentables, sont également insuffisantes.

C'est le contraire pour les Centers for Disease Control des États-Unis, où des effectifs bien formés et expérimentés font œuvre de pionniers pour contrer l'épidémie de VIH. En 2010, les États-Unis ont publié une stratégie impressionnante, assortie d'indicateurs d'impact objectifs et chiffrables et d'échéanciers. On prétend souvent que le rôle de l'Agence canadienne est limité parce que notre Constitution relègue la responsabilité de la santé aux provinces. En fait, aux États-Unis aussi la santé est principalement du ressort des États. Le gouvernement canadien a la responsabilité de diriger, de coordonner et de soutenir la lutte contre les problèmes de santé d'envergure nationale. Le VIH entre sûrement dans cette catégorie.

Nous devons mobiliser les meilleurs cerveaux du Canada à l'échelle fédérale. Il nous faut une équipe compétente et expérimentée de spécialistes de la santé publique, d'épidémiologistes, de spécialistes des sciences sociales et d'économistes pour élaborer et appuyer des politiques et des programmes novateurs et efficaces afin de contrer l'épidémie de VIH.

Robert Remis

Rédacteur scientifique intérimaire 